\title{
El estudio de la comunicación interna en el contexto del grado de publicidad y RR PP de la universidad de Cádiz
}

\author{
Pedro Pablo Marín DueÑas \\ Universidad de Cadiz \\ pablo.marin@uca.es \\ María GARCÍA GARCíA \\ Universidad de Cadiz \\ mariagarcia.garcia@uca.es
}

\begin{abstract}
Resumen
Frente a la creciente importancia y necesidad de comunicación con los públicos que tienen las organizaciones de todo tipo, la atención que las empresas prestan a la comunicación interna es todavía escasa. A nivel material la comunicación interna adolece de presupuestos poco consolidados y de personal poco cualificado para su gestión. Además, a nivel psicológico recibe escasa atención desde las direcciones de las organizaciones. Numerosos autores (Piñuel, 1997: 103; Villafañe, 1999: 301; Hernández, 1991: 268) han puesto de manifiesto la escasa importancia que le otorgan las empresas a este tipo de comunicación. Formar a universitarios competentes en este tipo de comunicación debería ayudar a solventar esta situación. De acuerdo con el Libro blanco de los títulos de grado en Ciencias de la Comunicación los estudiantes del grado de Publicidad y Relaciones Públicas, como gestores de la comunicación corporativa, serán capaces de gestionar la comunicación interna de la empresa correctamente.

En este trabajo se analiza el proceso de aprendizaje de los estudiantes del Grado en Publicidad y Relaciones Públicas de la Universidad de Cádiz desde el punto de vista de la formación en comunicación interna. De esta forma se reflexiona sobre el propio proceso de aprendizaje de la comunicación interna y se detectan carencias presentes que permitan prever insuficiencias futuras.

Para ello se eligió a los 119 alumnos de la asignatura de Sistemas de Comunicación Empresarial I a quienes se les encargó la tarea de desarrollar un plan de comunicación interna para pequeñas y medianas empresas creadas por ellos mismos en el que desarrollasen un análisis situacional, estrategias, objetivos, y herramientas para poder llevar a cabo la implementación real de dicho plan.

Los resultados muestran dificultades para definir los públicos internos de una organización que se traducen en objetivos y herramientas poco acordes a un plan de comunicación interna. Esta confusión podría justificar la escasa concienciación con la comunicación interna detectada
\end{abstract}

Palabras clave: comunicación interna, plan de comunicación, EEES, alumnos.

\section{The study of internal communication in the context of the degree of adverti- sing and PR at the University of Cádiz.}

\begin{abstract}
The importance and the need of communication with the stakeholders that organizations is growing but the attention that they pay to internal communication is still limited. Taking in consideration a material level, internal communication suffers from poorly consolidated budgets and unskilled employment for management. Also, on a psychological level, internal communication receives little attention from the head of the organizations. Many authors (Piñuel , 1997 : 103; Villafane, 1999 : 301; Hernandez, 1991 : 268 ) have shown the low importance given by companies to internal communication.
\end{abstract}


Training competent university students in this type of communication should help to resolve this situation. According to the White Book in Communication Sciences, Advertising and Public Relations the degree students, as corporate communication managers, should be able to manage the internal communication of the company correctly.

In this paper we analyse the learning process of the students of the degree in Advertising and Public Relations from the University of Cádiz, focusing in the internal communication training. Reflexing about this process, improvement will reflect on the learning process of internal communication and deficiencies detected can help to forecast future shortfalls.

119 students of the subject Business Communication Systems were chosen to this study. They were tasked to develop an internal communication plan for a small and medium sized businesses, created for themselves, in which they should carry out a situation analysis, strategies, goals, and tools to perform the plan.

The results show difficulties defining the organization's internal stakeholders that result in objectives and tools unsuitable for the internal communication plan. This confusion could justify the low awareness with internal communication detected

Key words: internal communication, communication plan, students, EHEA.

Referencia normalizada:

Marín Dueñas, P. P. y García García, M. (2013) El estudio de la comunicación interna en el contexto del grado de publicidad y RR PP de la universidad de Cádiz. Historia y Comunicación Social. Vol. $18 \mathrm{~N}^{\circ}$ Especial Octubre. Págs. 97-.108.

Sumario: 1. Evolución de la comunicación interna en el seno de las organizaciones. 2. Los estudiantes del Grado de Publicidad y Relaciones Públicas como gestores de la comunicación interna. 3. Objetivos. 4. Metodología. 5. Resultados. 6. Conclusiones. 7. Bibliografía..

\section{Evolución de la comunicación interna en el seno de las organizaciones}

\subsection{Aproximación al concepto de comunicación interna}

En la década de los 90 la comunicación interna ha sido entendida como un proceso en el cual se transmitía información, es decir, el feedback con el receptor era escaso o nulo. Así Rodríguez (1991: 32), definió la comunicación interna a principio como el

"conjunto de actividades efectuadas por cualquier organización para la creación y mantenimiento de buenas relaciones con y entre sus miembros, a través del uso de diferentes medios de comunicación que los mantengan informados, integrados y motivados para contribuir con su trabajo al logro de los objetivos organizacionales".

Así entendida, la comunicación interna fomenta las relaciones entre los miembros de la organización ya que asocia la sana comunicación entre los empleados con el desempeño laboral. En la medida en que los empleados se sientan escuchados y tomados en consideración pondrán mayor énfasis en el cumplimiento de los objetivos. Más recientemente Macías et al. (2013) le dan la vuelta a esta afirmación al indicar que si se le da la importancia debida a la comunicación interna, ésta ayudará a fomentar los lazos entre el mismo personal. De una forma u otra debe quedar claro 
que las empresas están formadas por personas que deben interactuar y donde pueden surgir problemas que la comunicación interna puede ayudar a detectar y mejorar.

Más allá de las relaciones personales entre los miembros de la organización, el auge del corporate y la concienciación por parte de las organizaciones de la necesidad de establecer políticas de comunicación activas, ha dado lugar a enfoques del concepto que ponen de manifiesto que a través de ella se genera un flujo de comunicación que fluye en todos los sentidos para informar y ser informado (Castillo, 2010).

Se puede decir, por tanto, que la comunicación interna hoy en día debe ser entendida como aquella que fluye desde y hasta los públicos internos de las organizaciones: empleados, accionistas y propietarios con el fin de aunar esfuerzos y conseguir los objetivos marcados por la organización.

\subsection{Situación actual de la comunicación interna}

En las primeras aproximaciones al concepto se puso de manifiesto la importancia de las personas en la comunicación interna. Los trabajadores y demás públicos internos serán quienes sustenten la comunicación en las organizaciones. Personas con diferentes inquietudes, personalidades, motivaciones, formación, etc, que en el contexto laboral se ven obligadas a comunicarse y a entenderse.

La comunicación entre estas personas es, por tanto, condición sine qua non para la buena marcha de la organización, convirtiendo así la gestión de la comunicación en un pilar básico de cualquier estrategia empresarial. A pesar de esto parece que las empresas siguen otorgándole un papel poco relevante a la gestión de la comunicación interna (Piñuel, 1997; Villafañe, 1999; Hernández, 1991).

Si tradicionalmente las organizaciones han prestado escasa atención a la comunicación de puertas para adentro, algunos estudios demuestran un creciente interés de las actividades comunicativas a nivel interno entre empleados y organización (Casti1lo, 2010).

En los últimos años el papel de la comunicación interna en la gestión de los negocios no ha parado de crecer, tal y como demuestra Álvarez (2007) al afirmar que

"las compañías líderes asumen que la auténtica comunicación engloba el conjunto de las actividades de una organización encaminadas hacia la obtención del éxito empresarial, traducido éste en beneficios económicos y desarrollo comunitario".

De la misma forma Rivera et al. (2005) entienden que la comunicación facilita las relaciones entre la alta gerencia y los empleados, articulando sus intereses, expectativas y necesidades, de modo que faciliten el desarrollo del talento humano y la optimización de la producción.

No solo la comunidad científica recoge esta incipiente inquietud por la comunicación empresarial en sus estudios, sino que los profesionales de la comunicación son también conscientes de esta tendencia tal y como demuestra el estudio realizado por la Asociación de Directivos de la Comunicación (Dircom) titulado "El estado de la 
Comunicación en España" del año 2010. En él los profesionales encuestados destacan "la importancia sobre la evolución de la responsabilidad social y la comunicación interna". De los 993 profesionales encuestados el 85\% respondió que la comunicación interna ha ganado "alcance en el último lustro". Este estudio y los profesionales encuestados confían en el ascenso de la importancia de la comunicación interna ya que el $78 \%$ opinan que la comunicación interna crecerá en importancia en las organizaciones en los años venideros, ganando peso en las estrategias de comunicación de las empresas.

Otros organismos como la Fundación EOI recalcan la importancia de la comunicación interna para las organizaciones. En el estudio sobre el modelo español de gestión y reporting de intangibles para un Dircom 2010 consideran que la comunicación interna es una de las "funciones mayoritariamente desarrolladas por los Dircoms en su quehacer diario", si bien reconocen que aún queda un largo camino por recorrer para la consolidación de la misma en el contexto de las organizaciones.

Ambos estudios ponen de manifiesto la necesidad embrionaria de formar a profesionales con conocimientos profundos sobre gestión de la Comunicación Interna (Matilla y Hernández, 2012) capaces de desenvolverse correctamente en un nuevo entorno laboral cada vez más complejo

\section{Los estudiantes del grado de Publicidad y Relaciones Públicas como gestores de la comunicación interna}

Si se atiende a lo expuesto en el Libro Blanco de los títulos de grado en Ciencias de la Comunicación (que englobaría los estudios de Publicidad y Relaciones Públicas, Comunicación Audiovisual y Periodismo), los graduados en Publicidad y Relaciones Públicas deben desarrollar la capacidad de gestionar la comunicación interna de la empresa. La completa formación de profesionales en comunicación implica "el acercamiento, desde la reflexión y el estudio, al quehacer comunicativo, entendido como el conjunto de sus diferentes acciones, especialmente publicitarias, de relaciones públicas, de comunicación, interna, promocional, etc.," como uno de los objetivos del título, de acuerdo siempre con el Libro Blanco.

A priori podría pensarse que la comunicación interna debe ser objeto de estudio exhaustivo en otros títulos, como el de comunicación audiovisual. Sin embargo, si se examina con detenimiento el Libro Blanco éste indica que "los profesionales que hayan cursado este grado serán capaces de planificar, analizar y valorar estrategias comunicativas y de comunicación en las organizaciones en sus dimensiones interna y externa, comercial y corporativa".

La comunicación interna debería ser, por tanto, objeto de estudio de los graduados en Publicidad y Relaciones Públicas. Queda claro ya que el estudio y la gestión de la comunicación interna se sitúa al mismo nivel que otros tipos de comunicación que tienen más presencia en el grado (como la comunicación comercial). 
Esta equiparación con la comunicación externa o corporativa no tiene un reflejo real en los planes de estudio de la mayoría de universidades españolas en las que se han encontrado asignaturas que responden a títulos como "Comunicación corporativa e institucional" pero no se han encontrado asignaturas de comunicación interna exclusivamente.

El caso que se presenta a continuación aborda el estudio de la comunicación interna en el grado de Publicidad y Relaciones Públicas en la Universidad de Cádiz donde, a falta de una asignatura específica y por coherencia, se eligió la asignatura de Sistemas de Comunicación Empresarial (cursada en el segundo año).

Dicha asignatura se imparte en los estudios de doble grado en Publicidad y Relaciones Públicas y en Marketing e Investigación de Mercado, doble grado en Publicidad y Relaciones Públicas y en Turismo y el grado en Publicidad y Relaciones Públicas, por lo que requiere de un enfoque transversal y multidisciplinar que aborde la comunicación empresarial en su sentido más amplio

\section{Objetivos}

El estudio realizado pretende analizar los conocimientos adquiridos por los estudiantes del grado de Publicidad y Relaciones Públicas de la Universidad de Cádiz en relación a la gestión de la comunicación interna. De esta forma se podrán detectar eventuales carencias y adaptar el estudio de la misma en los años venideros de forma que los estudiantes finalicen los estudios con los conocimientos y las capacidades suficientes sobre comunicación interna.

El objetivo del estudio es tener continuidad en el tiempo. De una parte puede servir para medir la calidad de las enseñanzas en comunicación interna que reciben los alumnos del grado en Publicidad y Relaciones Públicas de la Universidad de Cádiz. De otra, la reflexión y el análisis realizado permitirá realizar las modificaciones y las recomendaciones necesarias para formar a profesionales completos tal y como demanda el mercado laboral. La continuidad del estudio es importante para evaluar el proceso de formación de los alumnos a largo plazo, de acuerdo con la filosofía que conlleva el Plan Bolonia en el sentido más profundo de la reforma

\section{Metodología}

\subsection{Definición de la muestra y metodología empleada}

El objetivo de la investigación era detectar debilidades en la docencia y estudio de la comunicación interna en una carrera de comunicación de cara a reforzar los planes de estudio de los grados en comunicación así como reforzar la eficacia docente en esta área más que establecer generalizaciones estadísticas. 
Para ello, la muestra empleada en este estudio han sido los 119 alumnos matriculados en la asignatura de Sistemas de Comunicación Empresarial en la Universidad de Cádiz en el curso académico 2012/2013. El 80,67\% eran alumnos del grado de Publicidad y Relaciones Públicas (entre los que había 6 alumnos de diversos programas de movilidad); el 10,08\% cursaban los estudios del doble grado en Publicidad y Relaciones Públicas y Turismo mientras que el 9,24\% correspondían al grado en Publicidad y Relaciones Públicas.

Con el fin de obtener la información necesaria se ha recurrido a una técnica ampliamente aceptada en las ciencias sociales como es el análisis de contenidos. Según Berelson (1952) consiste en "la descripción objetiva, sistemática y cuantitativa del contenido manifiesto de la comunicación".

El análisis de contenidos se llevó a cabo gracias a la aplicación de un cuestionario elaborado a modo de checklist. Así se han revisado los 22 trabajos entregados pudiendo valorar el grado de conocimiento, desarrollo y comprensión de los conceptos y herramientas de la comunicación interna explicados en las clases teóricas y trabajados, con la supervisión del profesor, durante las clases prácticas.

\subsection{Plan de trabajo}

Para lograr la capacitación de los alumnos en el desarrollo de un plan de comunicación interna y alcanzar los objetivos planteados en la asignatura de Sistemas de Comunicación Empresarial I se planteó la realización de un trabajo en grupo ya que, de acuerdo con Marqués (2010), la realización de trabajos grupales permite:

- Realizar ejercicios sencillos conjuntamente.

- Realizar actividades de competición dentro del grupo.

- Que unos alumnos corrijan / ayuden a otros

- Exponer / compartir su trabajo con el grupo clase.

- Adoptar roles de profesor ante la clase.

- Resolver problemas complejos, crear conocimiento

Además, tal y como han demostrado estudios previos sobre metodología en el Espacio Europeo de Educación Superior, los alumnos muestran una mejor predisposición si asumen un rol activo y autónomo en el aula, limitando la función del profesor a la de guía y facilitador del aprendizaje (Vadillo, 2010).

Teniendo en cuenta lo anterior se les planteó a los alumnos la realización de una actividad práctica consistente en la elaboración de un plan de comunicación interna así como su implantación en una empresa ficticia (más concretamente en una pequeña y mediana empresa ) que fuese creada por los propios alumnos. De esta manera se facilitaba a los alumnos la simulación del entorno organizacional, pues son ellos los que iban a definir las características propias de su organización en cuanto a estructura, número de empleados, complejidad, etc. 
Otro de los motivos por los que se decidió que la empresa fuese ficticia fue porque en la asignatura de Diseño Gráfico, Multimedia y Espacios Comerciales, que cursan de forma simultánea, debían realizar un plan de comunicación externa para una empresa creada por ellos mismos, por lo que se les dio la posibilidad de utilizar la misma empresa para las dos asignaturas. De esta forma, se buscaba establecer sinergias entre las distintas asignaturas y proporcionar al alumno una visión global del proceso de comunicación en las organizaciones.

Para la ejecución de la actividad se dividió a los 119 alumnos matriculados en la asignatura en grupos formados por entre 5 y 6 estudiantes siendo finalmente 22 grupos de trabajo. Para facilitar la realización de la tarea se les entregó a cada uno de los grupos un documento con instrucciones detalladas sobre la actividad a realizar de forma que todos orientasen su esfuerzo en la misma dirección y que los requerimientos del trabajo para la posterior evaluación estuviesen claros desde el primer momento. llar:

En dicho documento de trabajo se contemplaban los siguientes puntos a desarro-

1. Diagnóstico de la situación actual (Presentación de la empresa (¿Quiénes somos?), Misión, visión y valores, Contexto del plan de comunicación interna (justificación, análisis DAFO de la situación).

2. Estrategias y objetivos para cada grupo de interés. Definir los públicos objetivos del plan de comunicación así como las estrategias a seguir para alcanzarlos.

3. Definir acciones y calendario.

4. Puesta en marcha del plan de comunicación. Esta fase era ficticia puesto que el plan no se implantó realmente.

5. Seguimiento y evaluación. Definir los indicadores y las herramientas con las que se mediría la eficacia y el alcance de las acciones desarrolladas.

Partiendo de estas instrucciones, los alumnos debían entregar un plan de comunicación interna que, necesariamente incluyese como acciones obligatorias:

- Un Manual de acogida (que incluyese una carta del director)

- Un Tablón de anuncios (físico o digital)

- Una Revista interna

Se optó por este sistema de trabajo para que los alumnos contasen con unas pautas claras desde el inicio de la actividad que, por un lado, facilitasen su trabajo y, por otro, permitiera una evaluación clara, sistemática y objetiva. 


\subsection{Puesta en práctica}

Con la implantación del EEES el profesor ha dejado de ser un mero transmisor de información que imparte una serie de conocimientos a los alumnos, que son meros oyentes, para convertirse en un orientador, un guía, un incentivador (Martín et al., 2012) que sigue un método activo de enseñanza.

El profesor respondiendo a las exigencias del nuevo EEES debe guiar el esfuerzo del alumno a lo largo de su proceso educativo, facilitar la adquisición y el desarrollo de competencias de los mismos (Herrero, 2012) así como evaluar a partir de un proceso sistemático y continuo. Así mismo, surge la necesidad de una evaluación objetiva y formativa que consiga el feedback de los alumnos y permita la mejora continua de los mismos.

De acuerdo con esto, el trabajo en las clases prácticas de la asignatura de Sistemas de Comunicación Empresarial I se centró en dar independencia a los alumnos para que desarrollasen el plan de comunicación, siguiendo el calendario fijado de antemano y tutorizando y apoyando a los alumnos cuando les surgiesen dudas, sin dejar al margen el apoyo de lecciones teóricas que apoyasen el trabajo práctico y que les permitiesen alcanzar los objetivos establecidos para superar el mismo.

\section{Resultados}

Los resultados obtenidos permiten dibujar unas líneas maestras sobre el estudio de la comunicación interna en el grado en Publicidad y Relaciones Públicas en la Universidad de Cádiz.

En primer lugar se puede afirmar que los alumnos de la asignatura de Sistemas de Comunicación Empresarial identifican claramente la identidad corporativa de una organización. Son capaces de concretar la misión y la visión de la entidad de forma concisa y coherente. En este estadio inicial pero crucial del desarrollo del trabajo se aprecia una leve tendencia de los estudiantes a formular la visión de la organización como objetivos de futuro. A pesar de lo cual puede decirse que se identifican claramente los pilares organizacionales que sostendrán el plan de comunicación.

La elaboración del plan de comunicación necesita de un contexto y unas circustancias que justifiquen su elaboración. La conexión entre el contexto inmediato de la empresa (su mercado, sus políticas de comunicación actuales, etc.) se antoja compleja para los estudiantes. Se ha detectado una tendencia a la parcelación del trabajo que les lleva a definir las partes del mismo como compartimentos estancos con escasa o nula conexión entre ellos. De esta forma, la justificación de la elaboración del plan así como el análisis que hacen de la situación muestra algunas incoherencias o desconexión con lo que es la identidad corporativa en sí. Esto se evidencia en la definición de los objetivos, que son fijados por los alumnos, en muchas ocasiones, sin tener en cuenta las carencias realmente detectadas en el DAFO que debían que realizar. 
De hecho, en la elaboración del DAFO se aprecia cierta dificultad para diferenciar entre debilidades y amenazas, así como entre fortalezas y oportunidades. La tarea encomendada exigía la elaboración de un DAFO aplicado al contexto de la comunicación en la empresa, siendo este uno de los principales aspectos en los que se ha tenido que poner énfasis en el proceso de tutorización por parte del profesorado.

Los públicos de interés y las estrategias establecidas en el plan de comunicación para alcanzarlos es una de las principales debilidades encontradas. Durante el proceso de tutorización del trabajo se observó una cierta dificultad para definir los públicos internos de la empresa que, si bien se aclaró de forma constante, ha dado lugar a públicos definidos de forma genérica y más bien teórica e, incluso, confusiones entre públicos internos y externos. Se entiende que este podría ser el origen de la ínfima relación entre estrategias, públicos y acciones detectado.

En lo que a la definición de las acciones y a su calendarización respecta, no se han detectado grandes problemas. Los estudiantes han definido y temporalizado las acciones de forma satisfactoria. Si bien, y en línea con esa estancación a la hora de desarrollar el trabajo, se detecta como algunos grupos no son capaces de definir acciones para alcanzar los objetivos que han planteado, sino que plantean una serie de herramientas de actuación sin considerar si son las más adecuadas para alcanzar los objetivos que han fijado previamente.

Los sistemas de evaluación del plan presentan algunas deficiencias. Se teoriza sobre la utilización de herramientas de forma adecuada (como encuestas de satisfacción, buzón de sugerencias u otras herramientas) pero hay una escasa relación entre los indicadores que determinarán la consecución o no de los objetivos y la evaluación de todas las acciones del plan. En general, se presentan instrumentos de valoración del plan de comunicación de una forma bastante genérica y conectadas con los objetivos de una forma algo tangencial.

La parte mejor valorada ha sido la materialización de las herramientas. Han ejecutado de forma muy satisfactoria tanto el tablón de anuncios como la revista interna y el manual de acogida exigido. La carta de bienvenida a la organización del director de la entidad también presenta índices de valoración muy elevados.

Otro aspecto a destacar positivamente es la inclusión en el plan de comunicación de herramientas alternativas a las propuestas con el fin de potenciar la comunicación interna a través de las nuevas tecnologías y que, de hecho, suponen cierta innovación para la mejora de los procesos de comunicación interna: la implantación de wikis o el uso de apps móviles y las redes sociales son un ejemplo de estas herramientas propuestas por los estudiantes.

\section{Conclusiones}

El análisis realizado ha permitido observar dos dimensiones importantes del proceso de aprendizaje universitario: 
- Por un lado las competencias reales adquiridas por los alumnos en relación a la comunicación interna

- De otro lado valorar la metodología docente empleada

De esta forma, se han podido alcanzar los objetivos planteados en este trabajo.

A nivel metodológico se ha detectado una división del conocimiento en ítems o cajones aislados que dificulta que los alumnos tengan una visión global del trabajo. Sería necesario evitar esta parcelación del trabajo y reforzar la unión entre las distintas partes del mismo bien modificando las dinámicas de trabajo en el aula o bien reforzándola con más ejemplos prácticos.

En relación a los conocimientos adquiridos se puede decir que los estudiantes han alcanzado los objetivos marcados en la actividad. Sin embargo, se han detectado algunas lagunas en los conocimientos adquiridos que sería necesario reforzar en futuros cursos académicos.

Partir de un posicionamiento de marca y de mercado más claro facilitaría a los alumnos establecer el contexto para el plan de comunicación. La falta de contextualización de la comunicación interna en relación a los otros tipos de comunicación dificulta un anclaje del plan de comunicación interna con respecto a la estrategia empresarial global.

Esta confusión para establecer el contexto se ve reflejada en la dificultad para realizar un análisis de la situación actual (DAFO) del plan de comunicación. Sería necesario reforzar el estudio de las amenazas, debilidades, fortalezas y oportunidades de una empresa en relación a la comunicación en general y con la comunicación interna en particular.

Un aspecto importante en la elaboración del plan es definir correctamente a quien va dirigido. Tener claro quiénes son los destinatarios del plan de comunicación es imprescindible para diseñar estrategias y herramientas que permitan alcanzarlos. La división de los públicos en función del tipo de comunicación emitida debe ser reforzada en el contexto de este grado puesto que los alumnos identifican perfectamente los públicos externos de una empresa pero no así los internos.

La continuidad del estudio permitiría completar la formación en comunicación interna de los estudiantes del grado de Publicidad y Relaciones Públicas de la Universidad de Cádiz. Para ello, se podría ampliar la muestra a otras asignaturas e incluso a otros grados afines.

Proyecto financiado por Junta de Andalucía, Gobierno de Extremadura y Fondos Feder. 


\section{Bibliografía}

Libros

BERELSON, B. (1952): Content Analysis in Comunication Research, Free Press: Glencoe.

CASTILLO, A. (2010): Introducción a las Relaciones Públicas, IIRP, España.

DIRCOM (2010): Estado de la comunicación en España 2010. Madrid, DIRCOM.

PIÑUEL, J.L. (1997): Teoría de la comunicación y gestión de las organizaciones, Ed. Síntesis, Madrid.

VILLAFAÑE, J. (1999): La gestión profesional de la imagen corporativa, Ed. Pirámide, Madrid.

Capítulos o artículos en libros o revistas en papel

ÁlVAREZ, J. (2007). Comunicación Interna, la Estrategia del Éxito. Razón y Palabra, 56(12), 1-7.

ARRIBAS, A. (2000). Comunicación en la empresa: la importancia de la información interna en la empresa. Revista Latina de comunicación social, Vol. 3, № 27, 1-5.

HERNÁNDEZ, R. (1991): Medios de comunicación en las organizaciones. En Fernández Collado, C.: La comunicación en las organizaciones, Trillas, México: 245-274

MACIAS, D.; PONCE, F.G.; ESCOBEDO, N.M.; BERUNEM, J. Y CUEVAS-CONTRERAS, T. (2013). Exploración en la comunicación interna como efecto competitivo. European Scientific Journal, vol 9, n¹7, pp. 52-65.

RIVERA, A.; ROJAS, L.; RAMIREZ, F. Y ÁLVAREZ, T. (2005). La comunicación como herramienta de gestión organizacional. Negotium, 1(2), 32-48.

RODRÍGUEZ, H. A. (1991). Hacia una definición de la comunicación organizacional. En Fernández Collado, C.: La comunicación en las organizaciones, Trillas, México, 29-34

VERČIČ, A.; VERČIČ, D.; Y SRIRAMESHC, K. (2012). Internal communication: Definition, parameters and the future. Public Relation Review, 38(2), 223-230.

Artículos en publicaciones web

ESCRIBANO, JJ. ET AL. (2008). La enseñanza y evaluación de competencias transversales. http://bioinfo.uib.es/ joemiro/TTrGrupo/EnsenyarTE.pdf

HERRERO MARTÍNEZ, R.; MARTÍN FERNÁNDEZ, M.A. y Quintero Ordóñez, B. (2012): Evaluación de competencias con actividades académicas interdisciplinares. Revista científica electrónica de Educación y Comunicación en la Sociedad del Conocimiento, 12 (1): 106-126. Disponible en: http://www.ugr.es/ sevimeco/ revistaeticanet/numero12/Articulos/Formato/articulo7.pdf Recuperado el 17 de julio de 2013.

MARTÍN PEÑA, M.L.; DÍAZ GARRIDO, E Y DEL BARRIO IZQUIERDO, L. (2012): Metodología docente y evaluación por competencias. Una experiencia en la materia Dirección de producción. Investigaciones europeas de dirección y 
economía de la empresa, Vol. 18, No 3, págs. 237-247. Disponible en: http:// dialnet.unirioja.es/servlet/articulo?codigo $=4009076$ Recuperado el 17 de julio de 2013.

PERE MARQUÉS (2010): Modelos didácticos de aprendizaje en grupo y aprendizaje colaborativo con TIC. Disponible en: http://www.peremarques.net/libro1/ trabajocolaborativocontic.pdf

MATILLA, K. y HERNÁNDEZ, S. (2012): Bolonia $2^{\circ}$ año: Los estudios universitarios de Relaciones Públicas en Cataluña (curso 2011-12). Revista Internacional de Relaciones Públicas, Vol. II, No 4, 247-276. Recuperado de http://revistarelacionespublicas.uma.es/index.php/re rrpp/article/view/117

VADILLO BENGOA, Nerea et al (2010): "Proceso de adaptación de los estudios de Comunicación al EEES. El caso de Aragón, una comunidad pionera”, en Revista Latina de Comunicación Social, 65. La Laguna (Tenerife): Universidad de La Laguna, páginas 187 a 203, recuperado el 17 de julio de 2013, de http://www. revistalatinacs.org/10/art/892_Zaragoza/14_Nerea.html DOI: 10.4185/RLCS-652010-892-187-203

\section{Los autores}

Pedro Pablo Marín Dueñas, Licenciado en Administración y Dirección de Empresas (2005), en Publicidad y Relaciones Públicas (2007). Actualmente es Becario de Investigación de la Junta de Andalucía para áreas deficitarias y se encuentra desarrollando su tesis doctoral en el campo de la comunicación empresarial que compagina con la docencia en la Universidad de Cádiz en el Departamento de Marketing y Comunicación. Ha colaborado en capítulos de la obra colectiva titulada "Nuevos movimientos sociales y comunicación corporativa: la revolución de la acción" (actualmente en proceso de edición) así como realizado una estancia de investigación en Bélgica, en la Universidad de Gante.

María García García es Doctora en Comunicación Audiovisual por la Universidad de Extremadura (2012). Actualmente es profesora sustituta interina en la Universidad de Cádiz, en el Departamento de Marketing y Comunicación, en el que imparte docencia en el grado de publicidad y relaciones públicas. Ha participado en diversos proyectos de investigación con financiación pública, tanto autonómicos como nacionales, y realizado diversas estancias docentes y de investigación pre doctoral en países como Portugal o Francia así como otras de carácter posdoctoral en Reino Unido o Ecuador. Ha publicado artículos en revistas nacionales como Área Abierta, Icono 14 o Revista Latina de Comunicación Social y en otras de ámbito internacional como Perspectivas en Ciencia da Información, OBS o Journal of Organisational and Transformational Social Change. Es coautora de los libros titulados "Los retos de la comunicación" y "Cómo investigar en comunicación” (actualmente en imprenta). 